$06,11,19$

\title{
Теплоемкость и тепловое расширение нанокомпозитов триглицинсульфат-пористое стекло
}

\author{
(C) Е.А. Михалева ${ }^{1}$, И.Н. Флёров ${ }^{1,2}$, А.В. Карташев ${ }^{1,3}$, М.В. Горев ${ }^{1,2}$, М.С. Молокеев ${ }^{1,2,4}$, \\ Л.Н. Коротков ${ }^{5}$, E. Rysiakiewicz-Pasek ${ }^{6}$ \\ ${ }^{1}$ Институт фризики им. Л.В. Киренского СО РАН, ФИЦ КНЦ СО РАН, \\ Красноярск, Россия \\ ${ }^{2}$ Институт инженерной физики и радиоэлектроники, Сибирский фредеральный Университет, \\ Красноярск, Россия \\ ${ }^{3}$ Красноярский государственный педагогический университет им. В.П. Астафьева, \\ Красноярск, Россия \\ ${ }^{4}$ Дальневосточный государственный университет путей сообщения, \\ Хабаровск, Россия \\ ${ }^{5}$ Воронежский государственный технический университет, \\ Воронеж, Россия \\ ${ }^{6}$ Division of Experimental Physics, Faculty of Fundamental Problems of Technology, \\ Wroclaw University of Science and Technology, \\ 50-370 Wroclaw, Poland \\ E-mail: katerina@iph.krasn.ru
}

(Поступила в Редакцию 1 февраля 2018 г.)

Впервые выполнены исследования влияния ограниченной геометрии на теплоемкость и тепловое расширение композитов триглицинсульфат(TGS)-боросиликатное стекло. Обнаружено уменьшение энтропии и температуры фазового перехода $P 2_{1} \leftrightarrow P 21 / m$ в компоненте TGS- при уменьшении диаметра пор стекольной матрицы, происходящее при неизменных значениях аномальных величин теплоемкости и коэффициента теплового расширения. Оценки свидетельствуют о небольшом эффекте внутреннего давления на барический коэффициент $d T_{c} / d p$ TGS в композитах.

Исследование выполнено при финансовой поддержке РФФИ в рамках научного проекта № 16-32-00092 мол_а.

DOI: 10.21883/FTT.2018.07.46118.027

\section{1. Введение}

Возрастающий интерес к композиционным материалам обусловлен рядом причин. Во-первых, и в основном, это связано с возможностью изучения материалов разной физической природы (сегнетоэлектриков, ферромагнетиков, сегнетоэластиков) в „особых условиях“, которые можно обеспечить только путем создания композита. Во-вторых, многие вещества обладают рядом „полезных“ характеристик, на основе которых основан широкий круг функциональных элементов, используемых в разных областях техники, и эффективность которых может быть значительно увеличена в композитном материале. Последнее обстоятельство подтверждается многочисленными экспериментальными исследованиями, которые показали сильное влияние размерных факторов на свойства активного компонента композита. То есть свойства вещества могут претерпевать значительные изменения при переходе от объемного монокристалла к микро/наноразмерным кристаллам, пленкам, керамикам, композитам [1].

Особенностями композитных материалов являются отсутствие химического взаимодействия компонентов и наличие четкой границы раздела кристаллических фаз.
Существует несколько путей формирования ферроидных и мультиферроидных композитов [2]. Самый распространенный способ - это механическое смешивание измельченных компонентов с последующим приготовлением керамических образцов. Процесс является достаточно трудоёмким, так как необходимо контролировать размер частиц и плотность керамических образцов, которые определяют степень упругого взаимодействия компонентов $[3,4]$. Не менее распространенный способ связан с приготовлением моно- и многослойных тонких гибридных пленок, например, на основе пьезоэлектрических и магнитных оксидов [1]. Такой метод позволяет заранее задавать размеры как частиц компонентов, так и толщину слоев. Однако взаимодействие компонентов при этом является „поверхностным“. Приготовление композитов путем внедрения веществ в пористую матрицу (пассивный компонент) приводит к более „объемному“ взаимодействию компонентов.

В качестве матричного каркаса часто используют прочные малодеформируемые материалы, например, боросиликатные стекла, $\mathrm{SiO}_{2}, \mathrm{Al}_{2} \mathrm{O}_{3}$ и др., в которых можно создавать поры строгой направленности или хаотичные с контролируемым диаметром от нескольких ангстрем до микрометров [1]. Многочисленные лите- 
ратурные данные свидетельствуют, что из ферроидных материалов наиболее успешно удается внедрять сегнетоэлектрики, из которых легко приготовить насыщенный водный раствор. Матрица пропитывается водным раствором и при последующей сушке сегнетоэлектрик кристаллизуется в порах, благодаря чему возникает достаточно жесткая связь между стенками пор и кристаллитами.

Несмотря на то, что круг сегнетоэлектриков, внедряемых в поры различных матриц, достаточно широкий: $\mathrm{NaNO}_{2}$ [5], $\mathrm{Na}_{1-x} \mathrm{~K}_{x} \mathrm{NO}_{2}$ [6], KDP [7,8], ADP [8], $\mathrm{NH}_{4} \mathrm{HSO}_{4}$ [9], TGS [10] и т.д., исследования очень часто были направлены на установление характера и степени влияния ограниченной геометрии (типа матрицы и размера пор) на диэлектрические свойства композитов $[5,6,8-17]$. В то же время теплофизические исследования композитов сегнетоэлектрик-матрица, позволяющие получать полезную информацию о таких важных характеристиках фазовых переходов, как энтропия, тепловое расширение, восприимчивость к высоким внешним давлениям и т.д., проводились эпизодически и только поисковыми методами $[5,7,8]$. И лишь недавно нами выполнены детальные исследования теплоемкости, упругой деформации, коэффициентов теплового расширения и фазовой диаграммы температура-давление для ряда нанокомпозитов $\mathrm{NH}_{4} \mathrm{HSO}_{4}$-боросиликатное стекло с размерами пор в интервале 46-320 nm [18]. Полученные результаты позволили установить степень и характер влияния размера пор на температуру, род, энтропию, деформацию, диэлектрическую проницаемость и барические коэффициенты при последовательных фазовых переходах $P-1 \leftrightarrow P c \leftrightarrow P 21$. Выполнен также анализ и сопоставление эффектов внутреннего, за счет упругого взаимодействия компонентов, и внешнего гидростатического давлений. Фазовые переходы в $\mathrm{NH}_{4} \mathrm{HSO}_{4}$ являются ярко выраженными превращениями первого $(P-1 \leftrightarrow P c)$ и второго $\left(P c \leftrightarrow P 2_{1}\right)$ рода. Несомненный интерес представляет исследование влияния ограниченной геометрии на сегнетоэлектрики, испытывающие фазовый переход, близкий к трикритической точке, при котором теплофизические свойства характеризуются значительными аномалиями, но отсутствует скрытая теплота перехода. Именно поэтому в качестве сегнетоэлектрического компонента нами выбран триглицинсульфат (TGS), в котором такого типа сегнетоэлектрический фазовый переход $\left(P 2_{1} \leftrightarrow P 2_{1} / m\right)$ реализуется при $T_{C}=321.4 \mathrm{~K}$ и сопровождается значительным изменением энтропии $\Delta S=6.78 \mathrm{~J} / \mathrm{mol} \cdot \mathrm{K}=0.815 R[19]$. При исследовании различных свойств внедренного в разного рода пористые матрицы TGS, за исключением теплофизических, были установлены следующие особенности $[10-13,15,17]$ : 1) во внедренном TGS сохраняется сегнетоэлектрическое состояние ниже $\left.T_{C} ; 2\right)$ обнаружена текстура TGS в порах матрицы $\left.\mathrm{Al}_{2} \mathrm{O}_{3} ; 3\right)$ уменьшение размера пор сопровождается увеличением объема элементарной ячейки TGS по сравнению с объемным монокристаллом; 4) наблюдалось значительное размытие фазового перехода; 5) немонотонное изменение температуры перехода с уменьшением размера пор $-T_{C}$ сначала растет, а начиная с $70 \mathrm{~nm}$, убывает и становится ниже, чем в объемном TGS [15].

В настоящей работе выполнены исследования теплоемкости и теплового расширения ряда композитов TGSборосиликатное стекло, а также керамического образца TGS, приготовленного из материала, внедряемого в матрицу. Установлен характер влияния ограниченной геометрии и размерных факторов на теплофизические характеристики фазового перехода в TGS.

\section{2. Образцы и методы исследования}

В дальнейшем будем использовать сокращенные обозначения для боросиликатных пористых стекол (PG) и композитов TGS-стекло (TGS + PG).

Перед началом работ по изготовлению композитов был выращен массивный объемный монокристалл TGS из водного раствора в максимально равновесных тепловых условиях. Из кристалла был приготовлен квазикерамический образец путем прессования мелкодисперсного порошка в виде таблетки без последующего обжига, на котором были выполнены те же теплофизические исследования, что и на композитах.

Характеризация TGS выполнена при комнатной температуре с помощью рентгеновского порошкового дифрактометра Bruker D8 ADVANCE (Cu-K $\alpha$ излучение). На рентгенограмме (рис. 1, $a$ ) не обнаружено каких-либо рефлексов, указывающих на присутствие в исходном кристалле посторонних фаз и примесей.

В качестве матриц были выбраны боросиликатные стекла со средним диаметром пор 46, 160 и $320 \mathrm{~nm}$. Образцы стекол имели вид прямоугольных пластин с размерами $\sim(10.0 \times 10.0 \times 0.5) \mathrm{mm}$. Сертификация образцов выполнена по методике, описанной ранее [20]. Пористость всех стекол была близка к 50\% (таблица).

Внедрение TGS в матрицу выполнялось путем погружения высушенных стекол в насыщенный водный раствор TGS при температуре $70^{\circ} \mathrm{C}$ и выдержки в течение $\sim(10-20) \mathrm{min}$. Последующее охлаждение до комнатной температуры проводилось с небольшой скоростью $(\sim 0.1 \mathrm{~K} / \mathrm{min})$. Заполненные стекла тщательно высушивались при $100^{\circ} \mathrm{C}$. После каждой сушки поверхность стекол очищалась путем механической полировки от остатков кристаллов, сформировавшихся в процессе кристаллизации и сушки. Цикл повторялся 5-8 раз. Степень заполнения оценивалась как отношение объемов внедренного TGS к объему пор (таблица). Массы исследуемых композитов близки и варьируются в пределах 79-86 mg.

Результаты уточнения модели структуры методом Ритвельда, выполненные для композитов TGS + PG46, TGS + PG160 и TGS + PG320, приведены на рис. $1, b, c, d$ и свидетельствуют о том, что в порах матрицы присутствуют кристаллы TGS без посторонних фаз и включений. Наличие двух гало на рентгенограммах связано с аморфным состоянием стекол. 

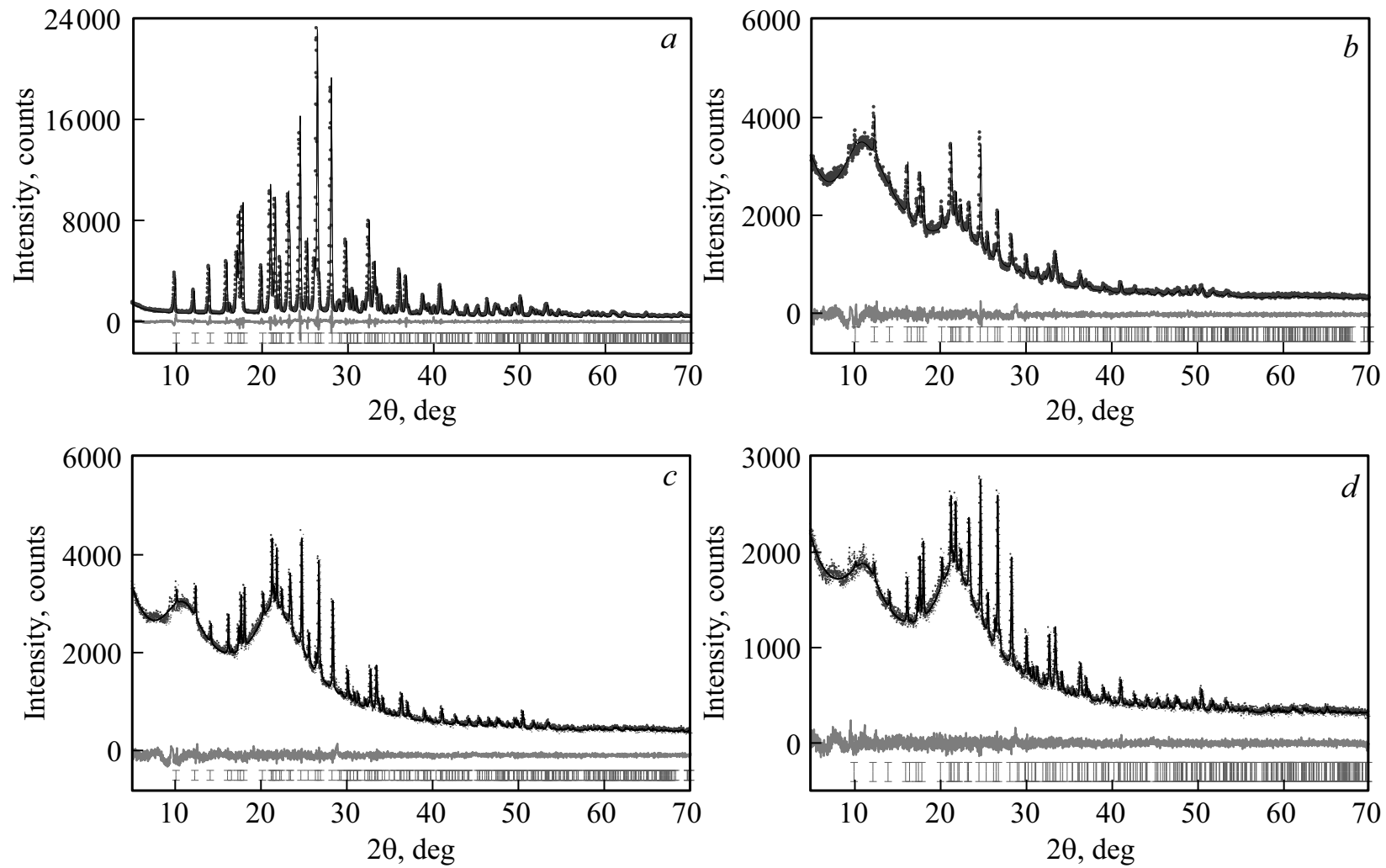

Рис. 1. Результаты уточнения модели структуры TGS (a) и для композитов TGS + PG46 (b), TGS + PG160 (c) и TGS + PG320 (d).

Анализ данных рентгеновских исследований с использованием программы TOPAS 4.2 [21] позволил определить размер кристаллитов $\left(d_{\text {cryst }}\right)$ в порах стекольной матрицы (таблица). Оказалось, что только в композите TGS + PG46 $d_{\text {cryst }}$ превышает диаметр пор $\left(d_{\text {pore }}\right)$, как это наблюдалось в ряде других исследований и объяснялось образованием дендритных кластеров [14,15]. Можно предполагать, что в случае TGS + PG160 и TGS + PG320 сегнетоэлектрик, не заполняя полностью объема пор, имеет надежный контакт с их стенками. В пользу этого свидетельствует тенденция, связанная с уменьшением объема элементарной ячейки $V_{\text {cell }}$ внедренного TGS по сравнению со свободным объемным кристаллом.

Следует отметить несущественное отличие параметров двух образцов TGS + PG320, приготовленных в независимых экспериментах, которое свидетельствует о воспроизводимости процессов заполнения матрицы сегнетоэлектрическим компонентом (таблица).

Исследование теплоёмкости $C_{p}(T)$ композитов и керамического TGS в широком интервале температур и вблизи фазового перехода выполнено на автоматизированном адиабатическом калориметре, использованном нами при изучении композитов $\mathrm{NH}_{4} \mathrm{HSO}_{4}$-стекло [18] и описанного в [22]. Эксперименты проводились в высоком вакууме $10^{-6} \mathrm{~mm} \mathrm{Hg}$ в режимах дискретных (с шагом $1-3 \mathrm{~K})$ и непрерывных $(d T / d t \approx 0.15-0.30 \mathrm{~K} / \mathrm{min})$ нагревов. Погрешность определения теплоемкости не превышала $0.5-1.0 \%$.

Температурные зависимости теплоемкостей нагревателя, контактной смазки и PG были определены в отдельных экспериментах. Ни в одном случае аномального поведения не обнаружено. Различие теплоемкостей всех исследованных стекол не превышает $2 \%$.

Некоторые параметры пористых матриц PG и композитов TGS + PG

\begin{tabular}{|c|c|c|c|c|}
\hline Размер пор $d_{\text {pore }}, \mathrm{nm}$ & $\begin{array}{c}\text { Пористость } \\
\text { стекол, \% }\end{array}$ & $\begin{array}{c}\text { Степень } \\
\text { заполнения, \% }\end{array}$ & $\begin{array}{c}\text { Размер } \\
\text { кристаллитов, nm }\end{array}$ & $\begin{array}{c}\text { Объем элементарной } \\
\text { ячейки TGS, nm }\end{array}$ \\
\hline $\begin{array}{r}46 \\
160 \\
320 \\
320 \\
\text { Объемный TGS }\end{array}$ & $\begin{array}{l}55 \\
46 \\
50 \\
50\end{array}$ & $\begin{array}{l}50 \\
61 \\
58 \\
48\end{array}$ & $\begin{array}{r}74(4) \\
133(4) \\
133(6) \\
168(6)\end{array}$ & $\begin{array}{l}0.64090(34) \\
0.64092(10) \\
0.64141(10) \\
0.641019(56) \\
0.642424(58)\end{array}$ \\
\hline
\end{tabular}



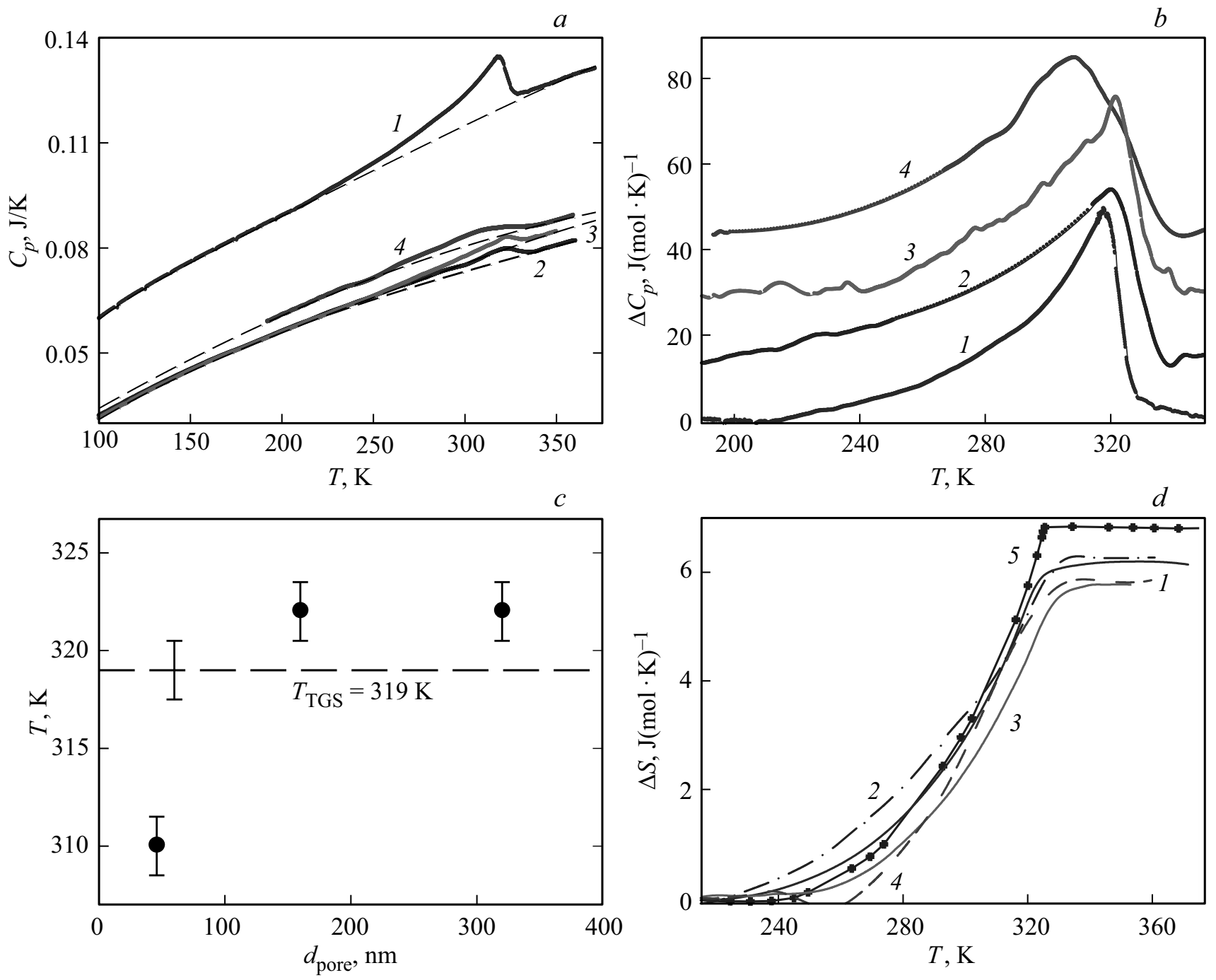

Рис. 2. Температурные зависимости теплоемкости керамического TGS (1), композитов TGS + PG320 (2), TGS + PG160 (3) и TGS + PG46 (4) $(a)$, аномальной теплоемкости $(b)$, энтропии фазового перехода $(d)$. На рисунке $(a)$ штриховые линии - регулярная составляющая теплоемкости. На рисунке $(b)$ кривые 2,3 и 4 сдвинуты вверх соответственно на 15,30 и $45 \mathrm{~J}(\mathrm{~mol} \cdot \mathrm{K})^{-1}$. Температуры фазовых переходов в композитах $(c)$. Данные об энтропии монокристаллического TGS (5) (рис. $d)$ заимствованы из [19].

Тепловое расширение исследовалось на дилатометре DIL-402C фирмы NETZSCH в температурном диапазоне $100-370 \mathrm{~K}$ в динамическом режиме со скоростью изменения температуры $3 \mathrm{~K} / \mathrm{min}$. Все измерения проводились в гелиевой атмосфере. Для калибровки и учета расширения измерительной системы использовались эталоны из плавленого кварца.

Предварительно выполненные измерения на стеклах показали как весьма малую величину коэффициента их теплового расширения, не превышающую $5 \cdot 10^{-6} \mathrm{~K}^{-1}$ в исследованном интервале температур, так и отсутствие аномального поведения. Вследствие большого отличия теплового расширения PG и TGS, ошибка определения соответствующих коэффициентов была существенной: $20 \%$ для PG и $8 \%$ для композитов. Однако расхождение данных, полученных в последовательных сериях измерений, не превышало $\sim 5 \%$.

\section{3. Экспериментальные результаты и обсуждение}

Результаты экспериментальных исследований теплоемкости композитов и керамического TGS представлены на рис. 2, $a$. На зависимостях $C(T)$ всех образцов отчетливо видна одна аномалия, относящаяся к фазовому переходу в TGS, которая размывается при уменьшении размера пор.

Для выделения аномального вклада $\Delta C_{p}(T)$ в теплоемкость сегнетоэлектрического компонента и определения интегральных характеристик фазового перехода теплоемкость композитов TGS + PG вдали от $T_{C}$ рассматривалась как регулярный вклад $C_{L A T}$. Результаты аппроксимации этих данных полиномиальной функцией показаны штриховой линией на рис. $2, a$. 
Экспериментальные температурные зависимости аномальной теплоемкости были пересчитаны в молярную теплоемкость TGS $\Delta C_{p}(T)$, которая представлена на рис. $2, b$. Видно, что температурный интервал наличия $\Delta C_{p}$ и ее величина мало меняются с изменением размера пор PG. Принимая температуру максимума $\Delta C_{p}$ за ТС, можно видеть, что фазовый переход в композитах TGS + PG320 и TGS + PG160 происходит при температуре, которая выше на $3 \mathrm{~K}$ по сравнению с $T_{C}=319.0 \pm 1.5 \mathrm{~K}$ для квазикерамического TGS, использованного для внедрения в матрицу (рис. 2,c). С другой стороны, при дальнейшем уменьшении $d_{\text {pore }}$ до $46 \mathrm{~nm} T_{C}$ уменьшается на $12 \mathrm{~K}$.

Путем интегрирования функций $\left(\Delta C_{p} / T\right)(T)$ определены изменения энтропии $\Delta S$, связанные с фазовым переходом в компоненте TGS, для всех исследованных в работе композитов TGS + PG, а также для квазикерамического TGS (рис. 2, $d$ ). Видно, что величины $\Delta S$ оказались меньше, чем энтропия, определенная для монокристалла $\Delta S_{\text {cryst }}=6.78 \mathrm{~J} / \mathrm{mol} \cdot \mathrm{K}[19]$. Максимальное различие (для композитов TGS + PG160 и TGS + PG46) составило $\sim 18 \%$, что больше ошибки измерения в экспериментах на композитах $( \pm 7 \%)$ и на монокристалле $( \pm 5 \%)$. Совпадение энтропий перехода для поликристаллического образца и композита TGS + PG320 объясняется, скорее всего, близостью размеров гранул и

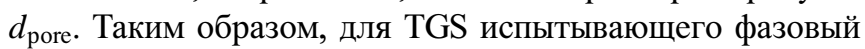
переход второго рода, близкий к трикритической точке, характерно небольшое уменьшение энтропии в условиях ограниченной геометрии, в отличие от композитов $\mathrm{NH}_{4} \mathrm{HSO}_{4}$-стекло, для которых уменьшение $d_{\text {pore }}$ в том же интервале приводило к уменьшению в полтора раза величины $\Delta S_{2}$, связанной с ярко выраженным переходом первого рода [18].

Температурные зависимости коэффициента линейного теплового расширения $\alpha$ для трех композитов TGS + PG и керамики TGS представлены на рис. 3. Можно видеть, что характер поведения $\alpha(T)$ композитов соответствует температурной зависимости коэффициента расширения керамического TGS. Абсолютные значения $\alpha$ композитов вне области перехода $\sim(0.9-1.2) \cdot 10^{-5} \mathrm{~K}^{-1}$ превышают $\alpha \approx(1-4) \cdot 10^{-6} \mathrm{~K}^{-1} \mathrm{PG}$ и, как и следовало ожидать, оказались меньше, чем $\alpha \approx(1.7-3.2) \cdot 10^{-5} \mathrm{~K}^{-1}$ для керамики TGS. Величины аномалий $\Delta \alpha$, связанных с фазовым переходом в компоненте TGS, уменьшились почти на порядок по сравнению с квазикерамическим образцом.

Температуры максимумов $\alpha(T)$, рассматриваемых в качестве $T_{C}$, удовлетворительно согласуются с температурами перехода, определенными из зависимостей $\Delta C_{p}(T)$. Таким образом, с одной стороны, фазовый переход в композитах TGS + PG320 и TGS + PG160 происходит при температуре несколько выше $T_{C}$ для квазикерамического TGS, а с другой стороны, $T_{C}$ значительно понижается при дальнейшем уменьшении $d_{\text {pore }}$ (рис. 2,c). Эти данные качественно согласуются с результатами, полученными при исследовании диэлектри-

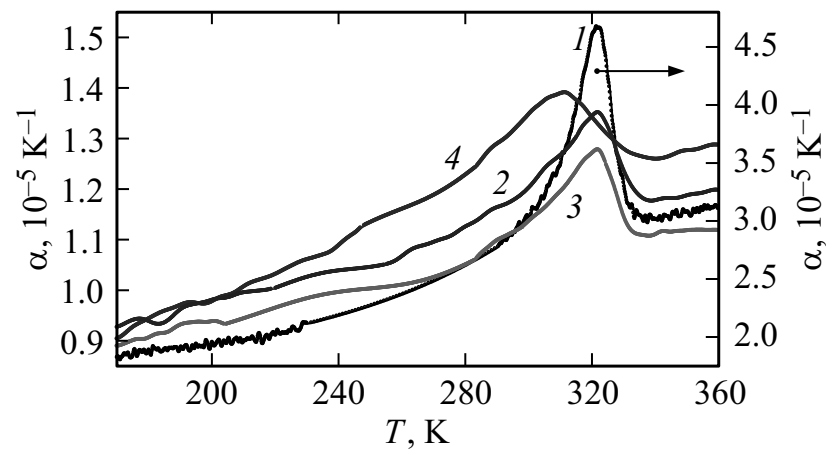

Рис. 3. Температурные зависимости коэффициента линейного теплового расширения керамического TGS (1) и композитов TGS + PG320 (2), TGS + PG160 (3) и TGS + PG46 (4).

ческой проницаемости ряда композитов TGS + PG [17]. Похожее поведение температур фазовых переходов наблюдалось и для композитов $\mathrm{NH}_{4} \mathrm{HSO}_{4}$-стекло [18].

Можно предполагать два механизма влияния размера пор на температуру фазового перехода в TGS, внедренного в стекольную матрицу. Один из них связан с большой разницей коэффициентов теплового расширения TGS и $\mathrm{PG}\left(\alpha_{\mathrm{TGS}} \gg \alpha_{\mathrm{PG}}\right)$, приводящей к возникновению растягивающих напряжений в сегнетоэлектрическом компоненте. Этот механизм, вероятно, играет основную роль в образцах с $d_{\text {cryst }}>100 \mathrm{~nm}$. В TGS температура перехода повышается с ростом давления $\left(d T_{C} / d p=2.6 \mathrm{~K} / \mathrm{kbar}\right)[23,24]$ и растягивающие напряжения должны бы приводить к понижению температуры перехода в композитах по сравнению с $T_{C}$ в квазикерамическом TGS. Однако небольшая величина барического коэффициента и малая разница температур заполнения стекла $(\sim 340 \mathrm{~K})$ и фазового перехода $(\sim 320 \mathrm{~K})$ в керамическом TGS не должны вызывать значительного изменения температуры сегнетоэлектрического перехода в композитах за счет этого эффекта. В то же время обнаруженное уменьшение объема элементарной ячейки внедренного TGS по сравнению со свободным объемным кристаллом (таблица) свидетельствует о наличии сжимающих напряжений, которые могут приводить к некоторому росту температуры перехода при $d T_{C} / d p>0$. Трудно отдать предпочтение одному из вариантов взаимодействия, так как повышение $T_{C}$ всего лишь на $\sim 3 \mathrm{~K}$ в композитах TGS + PG320 и TGS + PG160 сравнимо с суммарной ошибкой определения температуры довольно размытых аномалий теплоемкости и теплового расширения как в керамическом, так и в композитном образцах.

Другой механизм изменения температуры фазового перехода в сегнетоэлектрических наночастицах связан с изменениями баланса дальнодействующих и короткодействующих взаимодействий [25-27]. Можно с уверенностью считать, что именно этот механизм играет основную роль в композитах с малыми размерами частиц, $d_{\text {cryst }}<100 \mathrm{~nm}$, приводя к понижению $T_{C}$ при уменьшении $d_{\text {cryst }}$. 
Располагая данными о величинах $\Delta C_{p}$ и $\Delta \alpha$ при $T_{C}$, можно рассчитать сдвиг температуры фазового перехода второго рода под гидростатическим давлением, используя уравнение Эренфеста $d T / d p=T_{C}\left(\Delta \beta / \Delta C_{p}\right)$, где $\beta=3 \alpha$ для изотропных материалов - керамики и композитов. Рассчитанные для керамического образца и композита TGS + PG320 барические коэффициенты $d T / d p$ составили соответственно $6 \mathrm{~K} / \mathrm{kbar}$ и $0.9 \mathrm{~K} / \mathrm{kbar}$. Учитывая небольшой сдвиг температуры перехода под давлением можно считать удовлетворительным соотношение между рассчитанными $d T / d p$ и экспериментально измеренной величин $2.6 \mathrm{~K} / \mathrm{kbar}$ для монокристалла TGS [23]. Кстати, коэффициент расширения композитов определяется при следующем соотношении объема и его изменения с температурой $\beta=\left(1 / V_{\mathrm{TGS}+\mathrm{PG}}\right)\left(\Delta V_{\mathrm{TGS}+\mathrm{PG}} / \Delta T\right)$. Если же отнести изменение $\Delta V_{\mathrm{TGS}+\mathrm{PG}}$ к объему внедренного TGS, то величина $d T / d p=3 \mathrm{~K} / \mathrm{kbar}$ вполне удовлетворительно соотносится с экспериментальным значением.

\section{4. Заключение}

Впервые выполнены исследования влияния ограниченной геометрии на теплоемкость и тепловое расширение композитов TGS-боросиликатное стекло. Обнаружено немонотонное изменение температуры фазового перехода $P 2_{1} \leftrightarrow P 2_{1} / m$, сопровождающееся небольшим ростом в композитах TGS + PG320 и TGS + PG160 и значительным понижением в TGS + PG46, что качественно согласуется с данными [17]. Установлено незначительное изменение аномальных величин теплоемкости и коэффициента теплового расширения, связанных с фазовым переходом в TGS, при изменении диаметра пор стекольной матрицы. В большей мере происходит уменьшение энтропии перехода при уменьшении $d_{\text {pore }}$, однако даже в композите TGS + PG46 величина $\Delta S$ остается характерной для превращений типа порядок-беспорядок. Расчеты для композитов свидетельствуют о небольшом эффекте внутреннего давления на барический коэффициент.

Таким образом, теплофизические свойства при фазовом переходе второго рода в TGS, близком к трикритической точке, в значительно меньшей степени подвержены влиянию ограниченного пространства по сравнению со свойствами $\mathrm{NH}_{4} \mathrm{HSO}_{4}$ [18], испытывающего ярко выраженное превращения первого рода $P 1 \leftrightarrow P_{c}$.

Авторы признательны С.Д. Миловидовой за консультации по приготовлению композитов.

\section{Список литературы}

[1] Y. Kumzerov, S. Vakhrushev. Encyclopedia of Nanoscience and Nanotechnology 10, (2003). P. 1-39.

[2] М.И. Бичурин, В.М. Петров. Физика низких температур 36, 6, 680 (2010).
[3] E.A. Mikhaleva, I.N. Flerov, A.V. Kartashev, M.V. Gorev, A.V. Cherepakhin, K.A. Sablina, N.V. Mikhashonok, N.V. Volkov, A.V. Shabanov. J. Mater. Res. 28, 24, 3322 (2013).

[4] E. Mikhaleva, E. Eremin, I. Flerov, A. Kartashev, K. Sablina, N. Mikhashenok. J. Mater. Res. 30, 2, 278 (2015).

[5] Z. Kutnjak, B. Vodopivec, R. Blinc, A.V. Fokin, Y.A. Kumzerov, S.B. Vakhrushev. J. Chem. Phys. 123, 084708 (2005).

[6] С.Б. Вахрушев, И.В. Голосовский, Е.Ю. Королева, А.А. Набережнов, Н.М. Окунева, О.П. Смирнов, А.В. Фокин, M. Tovar, M. Glazman. ФTT 50, 1489 (2008).

[7] Ю.А. Кумзеров, Н.Ф. Картенко, Л.С. Парфеньев, И.А. Смирнов, А.В. Фокин, D. Wlosewicz, H. Misiorek, A. Jezowski. ФTT 53, 1033 (2011).

[8] V. Tarnavich, L. Korotkov, O. Karaeva, A. Naberezhnov, E. Rysiakiewicz-Pasek. Optica Applicata XL, 305 (2010).

[9] A. Ciżman, T. Marciniszyn, R. Poprawski. J. Appl. Phys. 112, 034104 (2012).

[10] O.V. Rogazinskaya, S.D. Milovidova, A.S. Sidorkin, N.G. Popravko, M.A. Bosykh, V.S. Enshina. Ferroelectrics 397, 191 (2010).

[11] N.G. Popravko, A.S. Sidorkin, S.D. Milovidova, O.V. Rogazinskaya. Ferroelectrics 443, 8 (2013).

[12] S.D. Milovidova, A.S. Sidorkin, O.V. Rogazinskaya, E.V. Vorotnikov. Ferroelectrics 497, 69 (2016).

[13] О.М. Голицына, С.Н. Дрождин, А.Е. Гриднев, В.В. Чернышев, И.Е. Занин. Изв. РАН. Сер. физ. 74, 9, 1347 (2010).

[14] A. Fokin, Yu. Kumzerov, E. Koroleva, A. Naberezhnov, O. Smirnov, M. Tovar, S. Vakhrushev, M. Glazman. J. Electroceram. 22, 270 (2009).

[15] О.М. Голицына, С.Н. Дрождин, И.Е. Занин, А.Е. Гриднев. ФTT 54, 2160 (2012).

[16] С.В. Барышников, Е.В. Чарная, Е.В. Стукова, А.Ю. Милинский, Cheng Tien. ФТТ 52, 1347 (2010).

[17] A. Cizman, T. Antropova, I. Anfimova, I. Drozdova, E. Rysiakiewicz-Pasek, E.B. Radojewska, R. Poprawski. J. Nanopart Res. 15, 1807 (2013).

[18] E.A. Mikhaleva, I.N. Flerov, A.V. Kartashev, M.V. Gorev, E.V. Bogdanov, V.S. Bondarev, L.N. Korotkov, E. Rysiakiewicz-Pasek. Ferroelectrics 513, 44 (2017).

[19] Б.А. Струков, Е.П. Рагула, С.В. Архангельская, И.В. Шнайдштейн. ФТТ 40, 106 (1998).

[20] A. Gutina, T. Antropova, E. Rysiakiewicz-Pasek, K. Virnik, Yu. Feldman. Micropor. Mesopor. Mater. 58, 237 (2003).

[21] Bruker AXS TOPAS V4, General profile and structure analysis software for powder diffraction data - User's Manual, Bruker AXS, Karlsruhe, Germany (2008).

[22] A.V. Kartashev, I.N. Flerov, N.V. Volkov, K.A. Sablina. Phys. Solid State 50, 2115 (2008).

[23] Г.Г. Леонидова, И.Н. Поландов, И.П. Голентовская. ФТТ 4, 3337 (1962).

[24] Y. Kobayashi, S. Sawada, H. Furuta, S. Endo, K. Deguchi. J. Phys.: Condens. Matter 14, 11139 (2002).

[25] R. Kretschmar, K. Binder. Phys. Rev. B 20, 1065 (1979).

[26] D.R. Tilley, B. Zeks. Solid State Commun. 49, 823 (1984).

[27] K. Ishikawa, K. Yoshikawa, N. Okada. Phys. Rev. B 37, 5852 (1988).

\section{Редактор Т.В. Василевская}

